\title{
Clinical experience with radio-, chemo- and hyperthermotherapy combined trimodality on locally advanced esophageal cancer
}

\author{
HAIWEN ZHU ${ }^{1}$, XUDONG HUO $^{1}$, LONGYUN CHEN ${ }^{1}$, HANHUA WANG $^{1}$ and HONGLIANG YU ${ }^{2}$ \\ ${ }^{1}$ Department of Radiation Oncology, Yancheng Third People's Hospital, Yancheng, Jiangsu 224000; \\ ${ }^{2}$ Department of Radiation Oncology, Jiangsu Cancer Hospital, Nanjing, Jiangsu 210000, P.R. China
}

Received January 2, 2013; Accepted May 8, 2013

DOI: $10.3892 / \mathrm{mco} .2013 .161$

\begin{abstract}
Esophageal cancer is a highly malignant and lethal disease with a low 5-year survival rate. Therefore, an effective treatment modality is required. To investigate the treatment efficacy and toxicity of radio-, chemo- and hyperthermotherapy combined trimodality on locally advanced esophageal cancer, the medical records of 78 patients with pathologically confirmed esophageal cancer treated with chemoradiotherapy plus hyperthemia at our institution were retrospectively investigated and the 3-year outcome was carefully assessed. All 78 patients received intensity-modulated radiation therapy at a total dose of 60-66 Gy, in a conventional schedule of 1.8-2.1 Gy/fraction, 5 fractions/week. They also received 4-6 courses of chemotherapy, consisting of $450 \mathrm{mg} / \mathrm{m}^{2} 5$-fluorouracil for $1-5$ days and $25 \mathrm{mg} / \mathrm{m}^{2}$ cisplatin for 1-5 days, in addition to 6-12 sessions of hyperthermia, performed twice a week. Out of the 78 cases, complete remission of the primary tumor was observed in 31 (39.7\%), partial remission in $44(56.4 \%)$ and no change in $3(3.9 \%)$ cases. The treatment response rate was $96.1 \%$. The overall survival (OS) rate at 1,2 and 3 years was $67.9,41.0$ and $33.3 \%$, respectively. No significant difference in adverse effects was observed between this treatment regimen and other similar studies. Our preliminary results demonstrated that the chemo-, radio- and hyperthermotherapy combined trimodality exhibited excellent short-term clinical outcomes as regards tumor response rate and a sound long-term OS, with endurable adverse events. This trimodal treatment requires further investigation to establish its beneficial role in the treatment of patients with locally advanced esophageal cancer.
\end{abstract}

Correspondence to: Dr Hongliang Yu, Department of Radiation Oncology, Jiangsu Cancer Hospital, No. 42, Baiziting Road, Nanjing, Jiangsu 210000, P.R. China

E-mail: yhllove1@163.com

Key words: esophageal cancer, radiotherapy, chemotherapy, hyperthermia, 3-year outcome

\section{Introduction}

Esophageal cancer is a highly malignant and lethal disease, with a particularly high incidence in China (1). To the best of our knowledge, a significant improvement of the 5-year survival rate was reported due to the advances in the therapeutic methods over the last 3 decades. However, its overall 5 -year survival remains low, at $\sim 19 \%(2,3)$. Therefore, an effective modality for the treatment of this malignancy is required.

Esophagectomy, with or without other adjuvant modalities, is preferred for patients with resectable esophageal cancer. For those patients who do not undergo surgical treatment for any reason, the Radiation Therapy Oncology Group (RTOG) trial 85-01 established concurrent chemoradiotherapy as the standard treatment (4). Hyperthermia is a modality that elevates tumor temperature to a supraphysiological level $\left(40-44^{\circ} \mathrm{C}\right)$, is a well-established radio- and chemosensitizer and widely accepted as an important adjuvant therapy to chemo- and radiotherapy (5). Hyperthermia, as part of a combination regimen, has demonstrated improved clinical response, local control and survival in numerous phase II studies and randomized trials in patients with breast, cervical, head and neck cancers, melanoma and glioblastoma multiforme (6-8). However, studies focusing on the application of hyperthermia on esophageal cancer treatment, which may be of interest to radiation oncologists, are limited. In this study, data on the combination of hyperthermia with chemo- and radiotherapy as a trimodal treatment for patients with locally advanced esophageal cancer are reported.

\section{Patients and methods}

Patients. A total of 78 patients hospitalized in our department between May, 2008 and December, 2009 were enrolled in the present study. Inclusion criteria were: pathologically diagnosed esophageal cancer, first-treated at our institution, no evidence of distant metastasis other than supraclavicular locoregional lymph nodes, Karnofsky Performance Status (KPS) $\geq 70$, no evidence of tracheoesophageal fistula and normal hepatic and renal function tests. This study was approved by the Institutional Review Board of Yancheng Third People's Hospital. Signed informed consent was provided by all the patients. 
Radiotherapy. All patients were immobilized within a thermoplastic mold and underwent CT stimulation. A total dose of 60-66 Gy was delivered to the $95 \%$ isodose line, which completely encompassed the planning target volume (PTV). A dose-volume histogram (DVH) was used to evaluate the dose received by PTV and adjacent critical tissue and organ. In the treatment plans, the dose variation in the PTV did not exceed $\pm 7 \%$, the dose received by the spinal cord was $>40 \mathrm{~Gy}$, the percentage of lung volume that received $20 \mathrm{~Gy}$ compared to the total lung volume (V20) was $\leq 25 \%$ and the mean dose to the heart was $<30 \mathrm{~Gy}$. The treatment plan was implemented following location and dose distribution verification, using 6-MV photons generated by a linear accelerator, in a normal delivery schedule of 1.8-2.1 Gy/fraction, 5 fractions/week. All treatment procedures were completed within 6-7 weeks.

Chemotherapy. All enrolled patients received 4-6 courses of monthly cycled concurrent cisplatin and 5-fluorouracil chemotherapy (PF therapy). Cisplatin $\left(25 \mathrm{mg} / \mathrm{m}^{2}\right)$ with hydration therapy was administered on days $1-5$ (at which time the radiotherapy was also initiated) and $450 \mathrm{mg} / \mathrm{m}^{2} 5$-flurouracil was infused intravenously on days 1-5. A total of 4-6 cycles of chemotherapy were administered, depending on the individual physical conditions of the patients.

Hyperthermia. Hyperthermia was applied by the BSD-2000 hyperthermia system (BSD Medical Corporation, Salt Lake City, UT, USA), which is able to heat locoregional lesions located deep in the body. Precise treatment planning was based on the lesion location as shown on the thoracic CT images, ensuring that the tumor was entirely located within the therapeutic thermal field. The objective of the hyperthermia treatment was to achieve an intratumoral temperature of $\geq 42.5^{\circ} \mathrm{C}$ for $60 \mathrm{~min}$. Hyperthermia was performed twice a week, within $2 \mathrm{~h}$ of the irradiation session during the period of radiotherapy. A total of 6-12 sessions of hyperthermia were performed, depending on the individual physical conditions of the patients.

Primary tumor response assessment. To evaluate the primary tumor response, thoracic CT scans, barium meal and ultrasound imaging were performed following the delivery of a dose of 40 Gy during the course of radiotherapy and at 3 and 6 months following the initiation of treatment. The treatment response was evaluated according to the revised RECIST guidelines (9).

Toxicity evaluation. Patients were carefully examined weekly throughout the duration of the treatment, or more often if clinically indicated. Patient physical profiles such as symptoms, physical signs, KPS and body weight were recorded in detail. Routine blood examination was performed weekly. A serum chemistry profile was performed prior to and following each chemotherapy cycle, to monitor hepatic and renal function. The toxicities were defined and graded according to the CTCAE, version 3.0 (10).

Statistical analysis. Descriptive results such as means, medians and proportions were calculated to characterize patient, disease and treatment characteristics, in addition to toxicities following treatment. The survival curves were estimated
Table I. Patient pretreatment characteristics.

Characteristics

No. of patients

Total patient no. 78

Age (years)

Mean

Range

Gender

Male

Female

Pathological type

Squamous cell carcinoma

Adenocarcinoma

Lesion site

Cervical

Upper thoracic 28

Middle thoracic 36

Lower thoracic 8

Primary lesion length $(\mathrm{cm})$

$\leq 5$

$>5$

Clinical stage

II

III

IV

using the Kaplan-Meier product-limit method (11). $\mathrm{P}<0.05$ was considered to indicate a statistically significant difference. Statistical software SPSS 19.0 (IBM SPSS Statistics, Chicago, IL, USA) was used for performing statistical analyses, manipulating data and generating data-summarizing graphs.

\section{Results}

Patient characteristics. As shown in Table I, the majority of the patients were male and the mean age was $65.1 \pm 5.2$ years. The predominant histological type was squamous cell carcinoma, with only one case of adenocarcinoma. The majority of cases were T3 or T4, N1 and M0 and clinical stage II or III.

Clinical primary tumor response. The clinical primary tumor response to the trimodal treatment was significant. Of the 78 patients enrolled in this study, 31 (39.7\%) exhibited a complete response (CR) of the primary lesions, 43 (56.4\%) exhibited a partial response (PR) and $3(3.9 \%)$ cases exhibited stable disease (SD). The total response rate $(\mathrm{CR}+\mathrm{PR})$ of the primary tumor to the trimodal treatment was as high as $96.1 \%$.

Disease control and survival. The mean follow-up duration was 20.1 months (range, 1.8-51.5 months). The clinical outcomes of locoregional control (LRC), distant metastasis-free survival (DMFS) and overall survival (OS) are shown in Table II. The 1-, 2- and 3-year LRC was 76.9, 55.1 and 47.4\%, respectively; the DMFS was $67.9,38.5$ and $30.8 \%$ respectively; 
Table II. OS, LRC and DMFS rates during the 3-year follow-up.

\begin{tabular}{lccc}
\hline Factors & 1-year (\%) & 2-year (\%) & 3-year (\%) \\
\hline OS & 67.9 & 41.0 & 33.3 \\
LRC & 76.9 & 55.1 & 47.4 \\
DMFS & 67.9 & 38.5 & 30.8 \\
\hline
\end{tabular}

OS, overall survival; LRC, locoregional control; DMFS, distant metastasis-free survival.

Table III. Adverse effects of the trimodality therapy.

\begin{tabular}{lrrrrr}
\hline & \multicolumn{5}{c}{ Grade } \\
\cline { 2 - 6 } Adverse effect & 0 & 1 & 2 & 3 & 4 \\
\hline Leucopenia & 4 & 12 & 30 & 30 & 2 \\
Thrombocytopenia & 57 & 9 & 6 & 6 & - \\
Anemia & 21 & 32 & 23 & 2 & - \\
Esophagitis & 13 & 31 & 26 & 8 & - \\
Nausea, vomiting & 68 & 6 & 3 & 1 & - \\
Pneumonitis & 76 & 2 & - & - & - \\
Liver dysfunction & 50 & 20 & 7 & 1 & - \\
\hline
\end{tabular}

with regards to OS, the median survival time was 24 months and the 1-, 2- and 3-year survival rate was 67.9, 41.0 and 33.3\%, respectively (Fig. 1). Compared to previous studies on concurrent chemoradiotherapy for esophageal cancer $(4,12,13)$, the OS outcome in our study was slightly improved. However, additional investigations are required to establish its statistical accuracy.

Treatment side-effects. The main toxicity of this trimodal treatment presented as hematological toxicity due to bone marrow suppression. Of the 78 follow-up patients, grade 3 or higher hematological side-effects included 32 cases of leucopenia $(41.0 \%), 6$ of thrombocytopenia $(7.7 \%)$ and 2 of anemia (2.6\%). Non-hematological toxicity mainly included radiation esophagitis, nausea or vomiting, pneumonitis and liver dysfunction (Table III). All patients succeeded in completing the treatment and no treatment-related mortality occurred within 90 days after the end of treatment. In general, the side-effects of the trimodal regimen were well-tolerated and no significant difference was observed between our therapy regimen and those of previous studies $(4,12)$.

\section{Discussion}

For patients with esophageal cancer, the efficacy of radiotherapy alone is not satisfactory, as demonstrated by the high incidence of treatment failure, presenting as locoregional tumor persistence or recurrence and distant metastasis. Over the past decades the medical community has endeavored to optimize treatment strategies, with the aim of reducing local and distant treatment failure. Concurrent chemoradiotherapy was

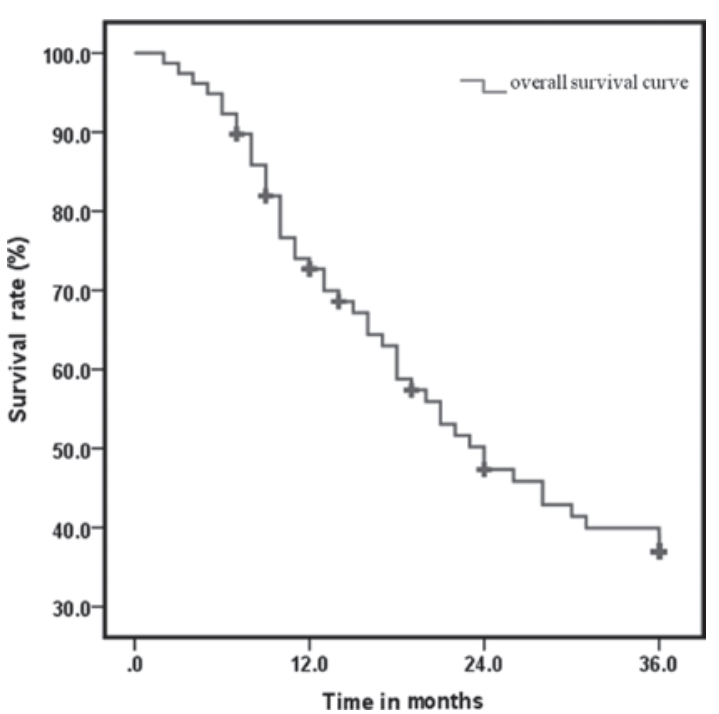

Figure 1. The overall survival curve of 3-year follow-up.

established as the standard treatment for esophageal cancer, as recommended by the RTOG trial 85-01 (4) in addition to other studies (2), with a notable improvement in treatment efficacy. However, disease persistence remains the most common cause of treatment failure and a significant predictor of worse OS (13). Therefore, improving LRC is critical in the treatment of esophageal cancer.

A trimodal treatment combining intensity-modulated radiation therapy (IMRT), chemotherapy and hyperthermia was applied at our institution to improve the LRC and ultimately the OS rates of esophageal cancer. IMRT is a product of the ongoing advances in radiotherapeutic technology. Compared to the conventional radiation dose-delivering technologies, IMRT has the ability to deliver higher doses to the tumor target, while limiting the irradiation of the surrounding normal tissues (14). Therefore, higher radiation doses may be delivered and an improved LRC may be achieved. With the use of this technology at our institution, the prescribed dose delivered to the PTV was 10-15 Gy higher than the dose used in the RTOG trial 85-01, with the aim of achieving optimal LRC benefits for the patients. With regards to the chemotherapy for esophageal cancer, concurrent administration of fluorouracil and cisplatin (PF therapy) was established as the standard adjuvant therapy and was applied at our institution. Locoregional hyperthermia is an additional modality for treating clinical malignancies, with no reported severe side-effects. The effect of hyperthermia on improving the local tumor control rate was significant and well-tolerated (6-8). Although hyperthermia alone may exert an antitumor effect, its synergistic effect with radiation and chemotherapy was the rationale for combining hyperthermia with chemoradiation therapy. Therefore, hyperthermia was included in the treatment regimen at our institution with the aim to improve the LCR of malignancies.

Our 3-year observation of the outcome of the trimodal treatment indicated a sound clinical efficacy. The primary tumor response rate was significant, reaching 96.1\%. This finding demonstrates the radiosensitizing effect of hyperthermia and chemotherapy and establishes the superiority of trimodal therapy. The clinical outcome of LRC, DMFS and 
OS in our study was satisfactory. The 1-, 2- and 3-year OS was $67.9,41.0$ and $33.3 \%$, respectively. Notably, compared with previous studies $(4,12,13)$, our preliminary results demonstrated that trimodal therapy exhibited a slightly improved long-term clinical outcome regarding the 3 -year OS $(33 \%$ in our study vs. $25 \%$ in chemoradiation therapy for locally advanced esophageal cancer), although additional investigations are required to verify this finding and establish its statistical accuracy. However, our results are similar to those reported by another study on the synergistic effect of hyperthermia and chemoradiation (15).

In our study, despite the higher radiation dose delivered and the application of hyperthermia, the toxicity was not significantly elevated. The most significant adverse effect of this regimen was hematological toxicity: $\geq$ grade 3 leucopenia was observed in $\sim 40 \%$ of the patients. However, there were no hematological toxicity-related mortalities, due to timely medical intervention and supportive care. There were no significant differences in the adverse effects between our trimodal treatment study and other similar studies $(4,12,16)$. Therefore, we concluded that the toxicity of the trimodal treatment was well-tolerated.

In conclusion, this study presents the single-institutional 3-year outcome of the radio-, chemo- and hyperthermotherapy combined trimodality on locally advanced esophageal cancer. The preliminary results have demonstrated that this regimen provides a good clinical outcome, presented by the high primary tumor response rate, as well as a slightly improved 3-year OS.

\section{References}

1. Jemal A, Center MM, DeSantis C and Ward EM: Global patterns of cancer incidence and mortality rates and trends. Cancer Epidemiol Biomarkers Prev 19: 1893-1907, 2010.

2. Kleinberg L, Gibson MK and Forastiere AA: Chemoradiotherapy for localized esophageal cancer: regimen selection and molecular mechanisms of radiosensitization. Nat Clin Pract Oncol 4: 282-294, 2007.
3. Jemal A, Siegel R, Xu J and Ward E: Cancer statistics. CA Cancer J Clin 60: 277-300, 2010.

4. Cooper JS, Guo MD, Herskovic A, et al: Chemoradiotherapy of locally advanced esophageal cancer: long-term follow-up of a prospective randomized trial (RTOG 85-01). Radiation Therapy Oncology Group. JAMA 281: 1623-1627, 1999.

5. Wust P, Hildebrandt B, Sreenivasa G, et al: Hyperthermia in combined treatment of cancer. Lancet Oncol 3: 487-497, 2002.

6. Franckena M, Lutgens LC, Koper PC, et al: Radiotherapy and hyperthermia for treatment of primary locally advanced cervix cancer: results in 378 patients. Int J Radiat Oncol Biol Phys 73: 242-250, 2009.

7. Moyer HR and Delman KA: The role of hyperthermia in optimizing tumor response to regional therapy. Int J Hyperthermia 24: 251-261, 2008.

8. Jones EL, Oleson JR, Prosnitz LR, et al: Randomized trial of hyperthermia and radiation for superficial tumors. J Clin Oncol 23: 3079-3085, 2005.

9. Eisenhauer EA, Therasse P, Bogaerts J, et al: New response evaluation criteria in solid tumours: revised RECIST guideline (version 1.1). Eur J Cancer 45: 228-247, 2009.

10. Trotti A, Colevas AD, Setser A, et al: CTCAE v3.0: development of a comprehensive grading system for the adverse effects of cancer treatment. Semin Radiat Oncol 13: 176-181, 2003.

11. Dinse GE and Lagakos SW: Nonparametric estimation of lifetime and disease onset distributions from incomplete observations. Biometrics 38: 921-932, 1982.

12. Minsky BD, Pajak TF, Ginsberg RJ, et al: INT 0123 (Radiation Therapy Oncology Group 94-05) phase III trial of combinedmodality therapy for esophageal cancer: high-dose versus standard-dose radiation therapy. J Clin Oncol 20: 1167-1174, 2002.

13. Zhang Z, Liao Z, Jin J, et al: Dose-response relationship in locoregional control for patients with stage II-III esophageal cancer treated with concurrent chemotherapy and radiotherapy. Int J Radiat Oncol Biol Phys 61: 656-664, 2005.

14. Galvin JM, Ezzell G, Eisbrauch A, et al: Implementing IMRT in clinical practice: a joint document of the American Society for Therapeutic Radiology and Oncology and the American Association of Physicists in Medicine. Int J Radiat Oncol Biol Phys 58: 1616-1634, 2004.

15. Kitamura K, Kuwano H, Watanabe M, et al: Prospective randomized study of hyperthermia combined with chemoradiotherapy for esophageal carcinoma. J Surg Oncol 60: 55-58, 1995.

16. Liu M, Shi X, Guo X, et al: Long-term outcome of irradiation with or without chemotherapy for esophageal squamous cell carcinoma: a final report on a prospective trial. Radiat Oncol 7: 142,2012 